\title{
Self-acceleration of a charge traveling into a waveguide
}

\author{
Andrey V. Tyukhtin* \\ Physical Faculty of Saint Petersburg State University, Saint Petersburg 198504, Russia
}

(Received 16 August 2013; published 26 February 2014)

\begin{abstract}
The interaction of a charge with its own electromagnetic field in the case of traveling into a circular semiinfinite waveguide is considered. The total work performed by the field on the charge is calculated analytically and presented as an integral over frequency. The typical spectral density of the work performed by the field is demonstrated. The total work depending on the velocity of the charge is computed. It is shown that the kinetic energy gain of the charge is positive if the velocity is less than a certain limiting value, which is independent of the waveguide radius.
\end{abstract}

DOI: 10.1103/PhysRevSTAB.17.021303

PACS numbers: 41.75.Lx, 41.60.-m

\section{INTRODUCTION}

As a rule, the fields produced by accelerated particles play negative roles in the acceleration process: they result in the deceleration of the particles and the instability of particle bunches. At the same time, self-fields can accelerate particles [1-5]. Such an effect cannot be realized if the charge environment at the end of the trajectory part under consideration is the same as the one at the beginning of the trajectory part. However, if the charge environment at the end of the considered trajectory segment differs from that at the beginning, then such a phenomenon can occur.

One example of such a situation is the case in which the charge intersects the border between two media $[1,5]$. Self-acceleration can also be achieved in vacuum structures [2-4]. In particular, Ref. [3] is devoted to the case in which a charge moves in an irregular waveguide that consists of an external infinite pipe with a half-infinite inner pipe. It was noted that the charge may experience an electromagnetic attracting force, but detailed analysis of this effect was not performed. It is known that the self-acceleration does not take place at ultrarelativistic velocities (in vacuum structures). However this effect is of essential importance to the fundamental understanding of the problem. Moreover, at present, this phenomenon may be of practical interest in connection with various medical and industrial applications that use not too rapid particles.

To explain the mechanism of self-acceleration, we write the energy conservation law in the following form:

$$
K^{(1)}+W^{(1)}=K^{(2)}+W^{(2)}+\Sigma,
$$

where $K^{(1)}$ and $W^{(1)}$ are, respectively, the kinetic energy of the charge and the energy of the electromagnetic field at the beginning of the trajectory segment under consideration,

\footnotetext{
*tyukhtin@bk.ru

Published by the American Physical Society under the terms of the Creative Commons Attribution 3.0 License. Further distribution of this work must maintain attribution to the author(s) and the published article's title, journal citation, and DOI.
}

$K^{(2)}$ and $W^{(2)}$ are the same values at the end of the trajectory segment, and $\Sigma$ is the total energy loss of the charge between points 1 and 2 . It is assumed that the system is passive, that is, $\Sigma \geq 0$. Note that in the absence of any ohmic losses, $\Sigma$ is radiation energy only.

The kinetic energy gain (KEG) is

$$
\Delta K=K^{(2)}-K^{(1)}=W^{(1)}-W^{(2)}-\Sigma .
$$

In the case when $W^{(1)}<W^{(2)}+\Sigma$, we have $\Delta K<0$; that is, the charge losses kinetic energy. However, if $W^{(1)}>W^{(2)}+\Sigma$, then $\Delta K>0$, that is, the charge is accelerated. Thus, the necessary and sufficient condition for self-acceleration is for the field energy difference $W^{(1)}-W^{(2)}$ to exceed the total losses $\Sigma$.

There is a simple qualitative explanation of the selfacceleration effect in the case of nonrelativistic motion of the charge. It is known that the energy of diffraction and transition radiation tends to zero for $\beta \rightarrow 0$ where $\beta=V / c$ ( $V$ is a charge velocity) $[1,6,7]$. Thus, for a nonrelativistic case, the sufficient condition for self-acceleration is $W^{(1)}>W^{(2)}$. The field energy is determined by the expression

$$
W^{(m)}=(8 \pi)^{-1} \int\left(\varepsilon_{m} \vec{E}_{m}^{2}+\mu_{m} \vec{H}_{m}^{2}\right) d V,
$$

where $\varepsilon_{m}, \mu_{m}$ are the permittivities and permeabilities, respectively, and $\vec{E}_{m}, \vec{H}_{m}$ are the electric and magnetic field strengths $(m=1,2)$. The integration in (3) is performed over the volume where the field exists. The inequality $W^{(1)}>W^{(2)}$ can be satisfied, for example, owing to differences in the permittivities or permeabilities $[1,5]$. In the case of vacuum structure (where $\varepsilon_{1}=\varepsilon_{2}=$ $\mu_{1}=\mu_{2}=1$ ), this inequality can be satisfied due to the difference in the conductive surfaces surrounding the charge trajectory, because these surfaces influence the electromagnetic field [2-4]. Naturally, for the case of relativistic motion, the radiation losses partially prevent self-acceleration. However, this effect must take place up to some limit value of the charge velocity while the radiation losses are small enough. 


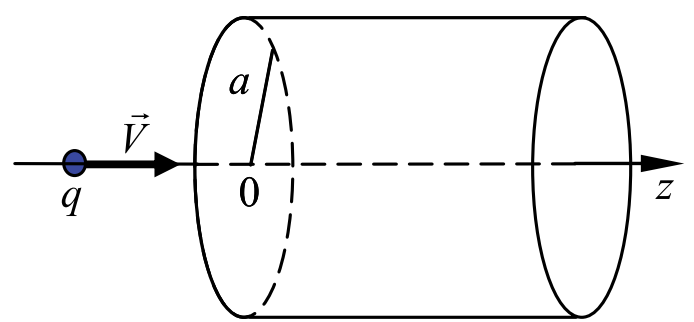

FIG. 1. The geometry of the problem.

In this paper, we consider a simple example of a vacuum structure that permits the self-acceleration effect: a circular semi-infinite $(z>0)$ waveguide of radius $a$ (Fig. 1). It is assumed that a point charge $q$ moves along the waveguide axis $z$ with velocity $\vec{V}=c \beta \vec{e}_{z}$, where $c$ is the speed of light in vacuum. We suppose that the velocity of the charge is essentially constant but the kinetic energy of the charge can vary. This assumption is exactly fulfilled only for a particle with infinite mass. However, this assumption is approximately true for many situations, in particular, for many problems of transition and diffraction radiation [1-10].

The KEG $\Delta K=K^{(2)}-K^{(1)}$ can be calculated using the conservation law (2). However, this method suffers from some difficulties. In particular, the energy of the field of a point charge is infinite; therefore, we would be obliged to consider some charge of finite size. It is easier and more natural to consider directly the force acting on the charge. In fact, the KEG is equal to the work performed by the field on the charge:

$$
\Delta K=A=\int_{-\infty}^{+\infty} F_{z} d z=\left.q V \int_{-\infty}^{+\infty} E_{z}\right|_{r=0, z=V t} d t .
$$

Thus, to find the KEG, we must solve this integral.

Note that the method based on the integral (4) is the traditional technique for calculation of the energy losses of a moving particle in different situations [1]. This method is equivalent to the calculation based on the energy conservation law (2). One of the important advantages of this method is that it requires calculation only of a single path integral, whereas the method based on the conservation law (2) requires finding both a volume integral $\left(W^{(1)}-W^{(2)}\right)$ and a surface integral $(\Sigma)$. Therefore, it is more convenient to use the obvious formula (4), as has been done for many other problems [1].

\section{SOLUTION OF THE BOUNDARY PROBLEM}

We write the total field $\left(\vec{E}^{\Sigma}, \vec{H}^{\Sigma}\right)$ in the form of the sum of the field of the charge in vacuum $\left(\vec{E}^{q}, \vec{H}^{q}\right)$ and some "additional" field $(\vec{E}, \vec{H})$ :

$$
\vec{E}^{\Sigma}=\vec{E}^{q}+\vec{E}, \quad \vec{H}^{\Sigma}=\vec{H}^{q}+\vec{H} .
$$

Using cylindrical coordinates, $r, \varphi, z$, the nonzero components of the "vacuum" field can be written in the form of the following Fourier integrals [10]:

$$
\begin{gathered}
\left\{E_{r}^{q}, E_{z}^{q}, H_{\varphi}^{q}\right\}=\int_{-\infty}^{\infty}\left\{E_{\omega r}^{q}, E_{\omega z}^{q}, H_{\omega \varphi}^{q}\right\} e^{-i \omega t} d \omega, \\
\left\{\begin{array}{c}
E_{\omega r}^{q} \\
E_{\omega z}^{q} \\
H_{\omega \varphi}^{q}
\end{array}\right\}=\frac{q}{\pi c^{2}}\left\{\begin{array}{l}
\frac{|\omega|}{\beta^{2} \gamma} K_{1}\left(\frac{|\omega| r}{V \gamma}\right) \\
\frac{-i \omega}{\beta^{2} \gamma} K_{0}\left(\frac{|\omega| r}{V \gamma}\right) \\
\frac{|\omega|}{\beta \gamma} K_{1}\left(\frac{|\omega| r}{V \gamma}\right)
\end{array}\right\} e^{i \omega z / V,}
\end{gathered}
$$

where $\gamma=1 / \sqrt{1-\beta^{2}}$, and $K_{0,1}(x)$ are the modified Hankel functions.

The "additional" field $\vec{E}, \vec{H}$ can be found using the Viner-Hopf-Fock technique, which has been applied to many problems of diffraction and diffraction radiation $[6,11,12]$. First, we write this field as a field of charge $\rho$ and current $\vec{j}$ induced on the waveguide wall. Because the wall is perfectly conductive and the magnetic field has only a $\phi$ component,

$$
\begin{aligned}
& \vec{j}=\vec{e}_{z} \delta(r-a) \int_{-\infty}^{\infty} \int_{-\infty}^{\infty} I\left(\omega, k_{z}\right) e^{i k_{z} z-i \omega t} d k_{z} d \omega, \\
& \rho=\delta(r-a) \int_{-\infty}^{\infty} \int_{-\infty}^{\infty} k_{z} \omega^{-1} I\left(\omega, k_{z}\right) e^{i k_{z} z-i \omega t} d k_{z} d \omega,
\end{aligned}
$$

where $I\left(\omega, k_{z}\right)$ is the Fourier transform of the surface density of the induced current (we take into account the charge-conservation law $\operatorname{div} \vec{j}+\partial \rho / \partial t=0$ ).

Based on known results concerning the field of an axially symmetric surface current (see, for example, [12]), one can obtain the following representation for the Fourier transform of the additional field:

$$
\begin{gathered}
E_{\omega r}=\frac{2 \pi^{2} i a}{\omega} \int_{-\infty}^{\infty} I k_{z} k_{r}\left\{\begin{array}{ll}
H_{0}^{(1)}\left(k_{r} a\right) J_{1}\left(k_{r} r\right) & \text { for } r<a \\
J_{0}\left(k_{r} a\right) H_{1}^{(1)}\left(k_{r} r\right) & \text { for } r>a
\end{array}\right\} e^{i k_{z} z} d k_{z}, \\
E_{\omega z}=-\frac{2 \pi^{2} a}{\omega} \int_{-\infty}^{\infty} I k_{r}^{2}\left\{\begin{array}{ll}
H_{0}^{(1)}\left(k_{r} a\right) J_{0}\left(k_{r} r\right) & \text { for } r<a \\
J_{0}\left(k_{r} a\right) H_{0}^{(1)}\left(k_{r} r\right) & \text { for } r>a
\end{array}\right\} e^{i k_{z} z} d k_{z}, \\
H_{\omega \phi}=\frac{2 \pi^{2} i a}{c} \int_{-\infty}^{\infty} I k_{r}\left\{\begin{array}{ll}
H_{0}^{(1)}\left(k_{r} a\right) J_{1}\left(k_{r} r\right) & \text { for } r<a \\
J_{0}\left(k_{r} a\right) H_{1}^{(1)}\left(k_{r} r\right) & \text { for } r>a
\end{array}\right\} e^{i k_{z} z} d k_{z},
\end{gathered}
$$


where $k_{r}=\sqrt{k^{2}-k_{z}^{2}}$, and $k$ is a wave number. Note that we have assumed that the medium is a vacuum, but we consider a vanishingly small imaginary part of $k$, that is, $k=\omega c^{-1}+i \delta$, where $\delta \ll|\omega| / c$ (small losses are taken into account). This standard approach allows us to define the radical $k_{r}=\sqrt{k^{2}-k_{z}^{2}}$ in a physically correcting way: $\operatorname{Im} k_{r}>0$ for all frequencies. Moreover, this approach helps us to clarify the relative positions of singularities and the integration path.

The boundary condition $\left.E_{z}\right|_{r=a, z>0}=-E_{z}^{q}$ results in the following integral equation:

$$
\begin{array}{r}
\int_{-\infty}^{\infty} I\left(k_{z}\right) \sqrt{a^{2} k^{2}-a^{2} k_{z}^{2}} \Phi\left(a k_{z}\right) e^{i k_{z} z} d k_{z} \\
=Q \exp (i \omega z / V) \text { for } z>0,
\end{array}
$$

where

$$
\begin{gathered}
\Phi(\xi)=\sqrt{a^{2} k^{2}-\xi^{2}} J_{0}\left(\sqrt{a^{2} k^{2}-\xi^{2}}\right) H_{0}^{(1)}\left(\sqrt{a^{2} k^{2}-\xi^{2}}\right), \\
Q=\frac{q a \omega^{2} H_{0}^{(1)}(i|\kappa|)}{4 \pi^{2} c^{2} \beta^{2} \gamma^{2}}, \quad \xi=a k_{z}, \quad \kappa=\omega a /(V \gamma)=k a /(\beta \gamma) .
\end{gathered}
$$

At the half-infinite cylindrical surface $r=a, z<0$, the component $H_{\phi}$ must be continuous; in other words, the induced current is absent here:

$$
\int_{-\infty}^{\infty} I\left(k_{z}\right) e^{i k_{z} z} d k_{z}=0 \quad \text { for } z<0
$$

Equations (9) and (11) form a system of paired integral equations. Such equations have been solved in [12] using the Viner-Hopf-Fock method for the case of the diffraction of a propagating wave at the end of the waveguide. Note that, in our problem, the projection of the incident wave onto the $z$ axis is larger than the vacuum wave number $(\omega / V>k=\omega / c)$. However this difference from the problem with a propagating incident wave does not impede one to use the technique described in [12].
With the aid of known results [12], the solution of Eq. (8) and (10) can be written in the following form:

$$
\begin{aligned}
I= & \frac{q \sqrt{1+\beta}(1-\beta) \omega^{3 / 2}}{8 \pi^{3} i V^{3 / 2} \Phi_{+}(\omega a / V)} \\
& \times \frac{H_{0}^{(1)}(i|\kappa|)}{\Phi_{-}\left(a k_{z}\right) \sqrt{k-k_{z}}\left(k_{z}-\omega V^{-1}-i 0\right)} .
\end{aligned}
$$

Here $\Phi_{ \pm}(\xi)$ are the factorizing functions for $\Phi(\xi)$, namely,

$$
\Phi_{+}(\xi) \Phi_{-}(\xi)=\Phi(\xi),
$$

where $\Phi_{+}(\xi)$ is holomorphic and does not have zeros in the region of $\operatorname{Im} \xi \geq 0$, and $\Phi_{-}(\xi)$ is holomorphic and does not have zeros in the region of $\operatorname{Im} \xi \leq 0$. These functions can be determined with the aid of the following formulas [12]:

$$
\ln \Phi_{ \pm}(\xi)= \pm \frac{1}{2 \pi i} \int_{\mp i \varepsilon-\infty}^{\mp i \varepsilon+\infty} \frac{\ln \Phi(\tilde{\xi})}{\tilde{\xi}-\xi} d \tilde{\xi}
$$

where $\varepsilon$ is some small positive value: $0<\varepsilon<\operatorname{Im}(k a)$. On the basis of (14), one can obtain representations in the form of infinite products [12], but we have no need of such representations for the following.

Thus we obtain the formal solution of the boundary problem that is given by Eqs. (8) and (12) with the factorizing functions (14).

\section{CALCULATION OF THE WORK PERFORMED BY THE FIELD}

The goal of this work is the calculation of the kinetic energy gain $\Delta K=K^{(2)}-K^{(1)}$, which is equal to the work (4) performed by the field on the charge. Note that the vacuum field (6) does not affect the charge, and only the additional field (8) makes a contribution in (4).

Naturally, the field components (8) are real. Therefore, $\vec{E}_{\omega}=\vec{E}_{-\omega}^{*}$ (the asterisk denotes complex conjugation), and we can transform the Fourier integral into an integral on the half axis:

$$
E_{z}=\int_{-\infty}^{\infty} E_{\omega z} \exp (-i \omega t) d \omega=2 \operatorname{Re} \int_{0}^{\infty} E_{\omega z} \exp (-i \omega t) d \omega .
$$

Using (8), (12), and (15), after some simple transformation, we obtain

$$
\left.E_{z}\right|_{r=0, z=V t}=\frac{q a}{\pi^{2} c^{3 / 2}} \frac{(1-\beta) \sqrt{1+\beta}}{\beta^{3 / 2}} \operatorname{Re}\left[\int_{0}^{\infty} d \omega \sqrt{\omega} K_{0}(\kappa) \int_{-\infty}^{\infty} \frac{G\left(t, \omega, k_{z}\right) d k_{z}}{k_{z}-\omega V^{-1}-i 0}\right],
$$

where

$$
G\left(t, \omega, k_{z}\right)=\frac{\left(k^{2}-k_{z}^{2}\right) H_{0}^{(1)}\left(a \sqrt{k^{2}-k_{z}^{2}}\right)}{\Phi_{+}(\omega a / V) \Phi_{-}\left(a k_{z}\right) \sqrt{k-k_{z}}} \times \exp \left[i\left(V k_{z}-\omega\right) t\right] .
$$


We first consider the inner integral in (16). Note that the integration pass is situated below the pole $k_{z}=\omega / V+i 0$. In the case of $t<0$, the function (17) is exponentially small for $\left|k_{z}\right| \rightarrow \infty$ if $\operatorname{Im} k_{z}<0$ because of the factor $\exp \left(i V t k_{z}\right)$. Therefore, we can lower the integration pass:

$$
\int_{-\infty}^{\infty} \frac{G\left(t, \omega, k_{z}\right)}{k_{z}-\omega V^{-1}-i 0} d k_{z}=\int_{-\infty-i \epsilon}^{\infty-i \epsilon} \frac{G\left(t, \omega, k_{z}\right)}{k_{z}-\omega V^{-1}} d k_{z} \text { for } t<0
$$

where $0<\varepsilon<\operatorname{Im} k$ (Fig. 2).

Analogously, for $t>0$, the function (17) is exponentially small for $\left|k_{z}\right| \rightarrow \infty$ if $\operatorname{Im} k_{z}>0$, and we can "lift" the integration pass higher on the real axis (Fig. 2). However, in this case, the pole $k_{z}=\omega / V+i 0$ is intersected by the

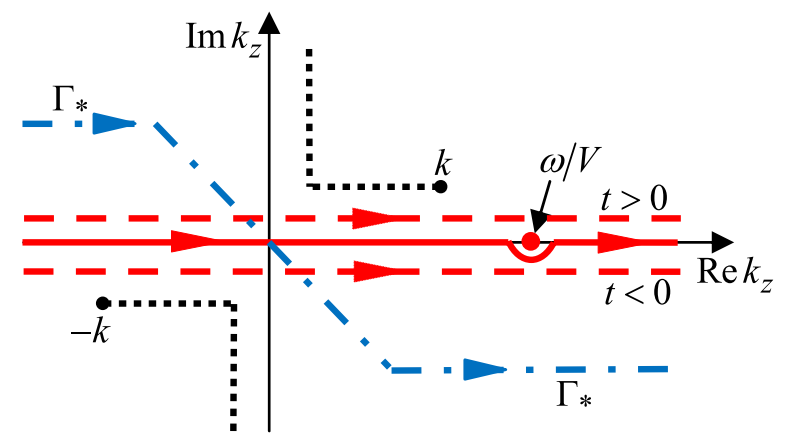

FIG. 2. The complex plane of $k_{z}$ : the cuts (dotted line), the initial integration path (solid line), the transformed integration paths in (21) (dashed lines), and the path $\Gamma_{*}$ in (23) and (24) (dash-dotted line).

contour transformation; therefore, we must take the corresponding residue into account. After some simple transformation, we obtain

$$
\int_{-\infty}^{\infty} \frac{G\left(t, \omega, k_{z}\right)}{k_{z}-\omega V^{-1}-i 0} d k_{z}=\int_{-\infty+i \varepsilon}^{\infty+i \varepsilon} \frac{G\left(t, \omega, k_{z}\right)}{k_{z}-\omega V^{-1}} d k_{z}+4 i \frac{\sqrt{1-\beta}(1+\beta)}{V^{3 / 2}} \frac{\omega^{3 / 2} K_{0}(\kappa)}{\Phi(\omega a / V)} \quad \text { for } t>0 .
$$

As we can see, the second summand in (19) (the pole contribution) is imaginary. Therefore, it does not contribute in (16), and we obtain, for arbitrary time,

$$
E_{z}=\frac{q a(1-\beta) \sqrt{1+\beta}}{\pi^{2} c^{3 / 2} \beta^{3 / 2}} \int_{0}^{\infty} d \omega \sqrt{\omega} K_{0}(\kappa) \int_{-\infty+i \varepsilon \operatorname{sgn} t}^{\infty+i \varepsilon \operatorname{sgn} t} \frac{G\left(t, \omega, k_{z}\right)}{k_{z}-\omega V^{-1}} d k_{z}
$$

We substitute (20) into (4) and present the result as a sum of integrals over negative and positive times:

$$
A=\frac{q^{2} a(1-\beta) \sqrt{1+\beta}}{\pi^{2} \sqrt{c \beta}} \int_{0}^{\infty} d \omega \sqrt{\omega} K_{0}(\kappa) \times \operatorname{Re}\left[\int_{-\infty}^{0} d t \int_{-\infty-i \varepsilon}^{\infty-i \varepsilon} \frac{G\left(t, \omega, k_{z}\right)}{k_{z}-\omega V^{-1}} d k_{z}+\int_{0}^{\infty} d t \int_{-\infty+i \varepsilon}^{\infty+i \varepsilon} \frac{G\left(t, \omega, k_{z}\right)}{k_{z}-\omega V^{-1}} d k_{z}\right] .
$$

Note that the function $\exp \left[i\left(V k_{z}-\omega\right) t\right]$, that is present in $G\left(t, \omega, k_{z}\right)$, is exponentially small for $t \rightarrow-\infty$ in the first summand in (21) and for $t \rightarrow+\infty$ in the second summand [because $\operatorname{Im}\left(t k_{z}\right)>0$ on the integration passes]. Therefore, we can easily integrate over time in (21) and obtain the following result:

$$
\begin{aligned}
A= & \frac{q^{2} a(1-\beta) \sqrt{1+\beta}}{\pi^{2} c^{3 / 2} \beta^{3 / 2}} \int_{0}^{\infty} d \omega \sqrt{\omega} K_{0}(\omega) \\
& \times \operatorname{Re}\left[i \int_{\infty-i \varepsilon}^{-\infty-i \varepsilon} \frac{G\left(0, \omega, k_{z}\right) d k_{z}}{\left(k_{z}-\omega V^{-1}\right)^{2}}+i \int_{-\infty+i \varepsilon}^{\infty+i \varepsilon} \frac{G\left(0, \omega, k_{z}\right) d k_{z}}{\left(k_{z}-\omega V^{-1}\right)^{2}}\right] .
\end{aligned}
$$

Note that here, the integrand decreases as $O\left(k_{z}^{-3 / 2}\right)$ if $\left|k_{z}\right| \rightarrow \infty$. Therefore, we can construct a closed integration path consisting of the infinity rectilinear parts $(-\infty+i \varepsilon, \infty+i \varepsilon)$ and $(\infty-i \varepsilon,-\infty-i \varepsilon)$ as well as from the infinitesimal segments $(\infty+i \varepsilon, \infty-i \varepsilon)$ and $(-\infty-i \varepsilon,-\infty+i \varepsilon)$. This contour includes only the single pole $k_{z}=\omega V^{-1}$ of the second order; therefore, the quantity contained in the square brackets in (22) can be written as $[\ldots]=2 \pi d G\left(0, \omega, k_{z}\right) /\left.d k_{z}\right|_{k_{z}=\omega / V}$. After some cumbersome calculations, we obtain

$$
A=\int_{0}^{\infty} \frac{d A}{d \omega} d \omega=\int_{0}^{\infty} \frac{d A}{d \tilde{k}} d \tilde{k}
$$

where $\tilde{k}=k a=\omega a / c$, and the spectral density of the work performed by the field is 


$$
\frac{d A}{d \tilde{k}}=\frac{q^{2}}{\pi a \beta I_{0}(\kappa)}\left\{(3-\beta) K_{0}(\kappa)-2 \kappa\left[K_{1}(\kappa)+\left.\frac{K_{0}(\kappa)}{\gamma} \operatorname{Re}\left(\frac{d \ln \Phi_{-}(x)}{d x}\right)\right|_{x=\tilde{k} / \beta}\right]\right\}
$$

Using (14), one can transform the derivative that appears in (24) in the following way:

$$
\begin{aligned}
\left.\frac{d \ln \Phi_{-}(x)}{d x}\right|_{x=\tilde{k} / \beta} & =-\frac{1}{2 \pi i} \int_{i 0-\infty}^{i 0+\infty} \frac{\ln \Phi(\xi)}{(\xi-\tilde{k} / \beta)^{2}} d \xi=\left.\frac{d \ln \Phi(x)}{d x}\right|_{x=\tilde{k} / \beta}-\frac{1}{2 \pi i} \int_{\Gamma_{*}} \frac{\ln \Phi(\xi)}{(\xi-\tilde{k} / \beta)^{2}} d \xi \\
& =\gamma\left[\frac{I_{1}(\kappa)}{I_{0}(\kappa)}-\frac{K_{1}(\kappa)}{K_{0}(\kappa)}+\frac{1}{\kappa}\right]-\frac{1}{2 \pi i} \int_{\Gamma_{*}} \frac{\ln \Phi(\xi)}{(\xi-\tilde{k} / \beta)^{2}} d \xi,
\end{aligned}
$$

where $\Gamma_{*}$ can be placed arbitrarily in the second and fourth quadrants (one variant of this contour is shown in Fig. 2). Substituting (25) into (24), we obtain

$$
\frac{d A}{d \tilde{k}}=\frac{q^{2} K_{0}(\kappa)}{\pi a \beta I_{0}(\kappa)}\left[1-\beta-2 \kappa \frac{I_{1}(\kappa)}{I_{0}(\kappa)}+\frac{\kappa}{\pi \gamma} \operatorname{Im} \int_{\Gamma_{*}} \frac{\ln \Phi(\xi) d \xi}{(\xi-k a / \beta)^{2}}\right] .
$$

We recall that $\kappa=\omega a /(V \gamma)=\tilde{k} /(\beta \gamma)$ and $\gamma=\left(1-\beta^{2}\right)^{-1 / 2}$.

\section{COMPUTATIONS AND DISCUSSION}

Expression (26) results in the following approximation for the case of $\kappa \ll 1$ :

$$
\frac{d A}{d \tilde{k}} \approx \frac{q^{2}(1-\beta)}{\pi a \beta}\left[\ln \left(\frac{2}{\kappa}\right)-C\right],
$$

where $C=0.577 \ldots$ is the Euler constant. Therefore, the spectral density of the KEG has a logarithmic singularity at $\kappa \rightarrow 0$. It is important that the value (27) is positive, i.e., the effect of the low-frequency part of the field must lead to the acceleration of the charge.

The asymptote for the case of $\kappa \gg 1$ has the following form:

$$
\frac{d A}{d \tilde{k}}=-\frac{2 q^{2} \kappa e^{-2 \kappa}}{a \beta}\left[1+\frac{1}{2 \pi \gamma} \operatorname{Im} \int_{\Gamma_{*}} \frac{\ln \Phi(\xi) d \xi}{(\xi-k a / \beta)^{2}}\right] .
$$

One can see that the spectral density of the work performed by the field takes an exponentially small value for high frequencies.

Representative results of the computation of the KEG spectral density from formula (26) are shown in Fig. 3. For sufficiently small charge velocities (top graphic), the lowfrequency positive part of these dependencies is dominant, and the negative part is negligible. An increase in $\beta$ results in a decrease in the positive part of the curve and an increase in the negative part that can be observed at higher frequencies (Fig. 3, middle graphics). Finally, the negative part becomes dominant in comparison with the positive one (Fig. 3, bottom graphics).

The total KEG (the total work of the field) as computed from the exact formulas (23) and (26) is shown in Fig. 4 as a function of the velocity of the charge. One can see that this dependence is monotonically increasing. The KEG is maximum if $\beta \rightarrow 0$. Computation yields the following value:

$$
\left.A\right|_{\beta \rightarrow 0} \rightarrow A_{\max } \approx 0.436 \times \frac{q^{2}}{a} .
$$
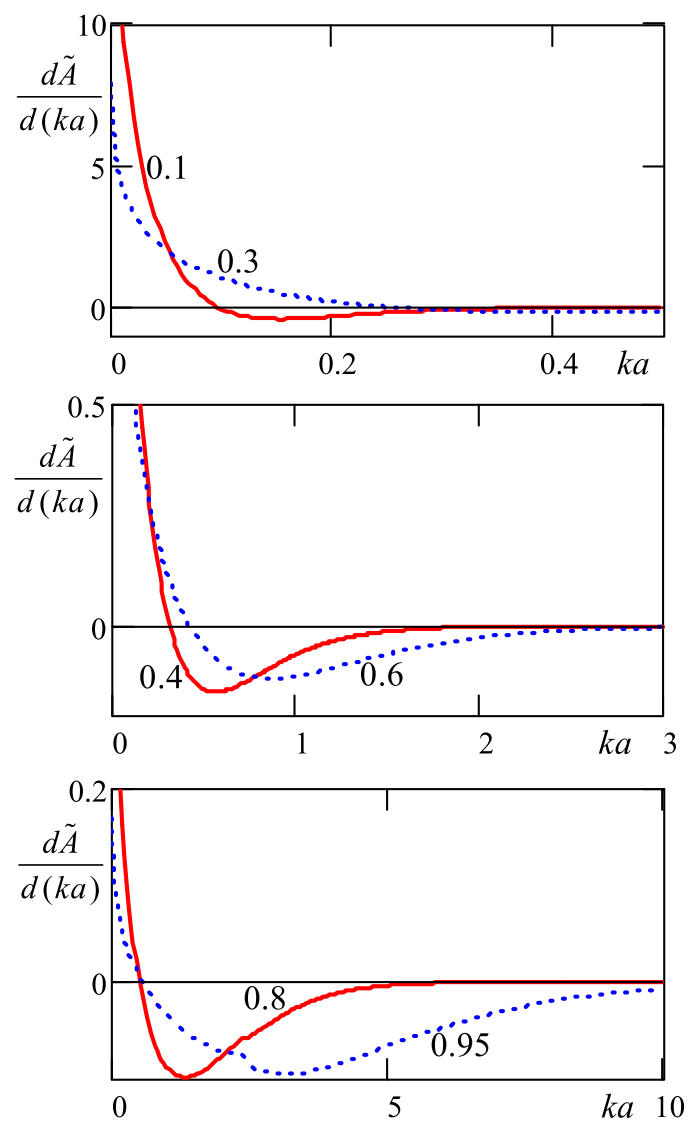

FIG. 3. The normalized spectral density of the work performed by the field, $d \tilde{A} / d(k a)=q^{-2} a d \tilde{A} / d(k a)$, depending on $k a=$ $\omega a / c$ for the different values of $\beta$ that are indicated near the curves. 


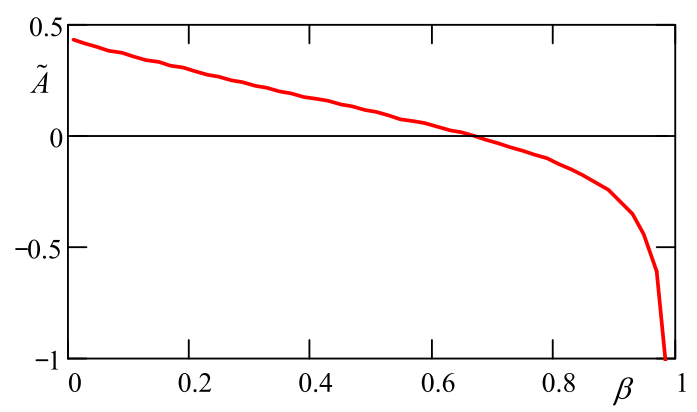

FIG. 4. The normalized work performed by the field $\tilde{A}=q^{-2} a A$, depending on the velocity of the charge.

One can also find that the KEG is positive for

$$
\beta<\beta_{\lim } \approx 0.663 \text {. }
$$

If $\beta>\beta_{\text {lim }}$, then the KEG is negative; that is, the charge loses kinetic energy. It is interesting that the limiting velocity $\beta_{\lim } \approx 0.663$ does not depend on the radius of the waveguide; however, the value of $A_{\max }$ varies inversely to the radius.

We have obtained the KEG for a charge flying from $-\infty$ to $+\infty$. It is interesting to find a real charge path required for obtaining this self-acceleration effect. Here, we give only a rough estimate of the minimal length of this path. We use the concept of a radiation formation length $l$ [1]. This value determines a path on which transitional processes take place. The rough estimate of this value is $l \sim V / \omega$ [6]. The path of the charge in the waveguide must be more than $l$ for the frequencies that mainly determine the KEG. These frequencies can be estimated from (27) as $\omega \sim V \gamma / a$, and we obtain $l \sim a / \gamma$. Because $\gamma \sim 1$, the self-acceleration effect is achieved for a charge path of the order of the waveguide radius.

The upper estimate of the KEG for electrons or protons can be obtained using (29). For a small bunch of $N$ particles with charge $e$, the maximum value of the KEG per particle $(\delta K=A / N)$ is

$$
\delta K_{\max }=\frac{A_{\max }}{N} \approx 0.436 \times \frac{N e^{2}}{a} \approx 0.628 \times 10^{-7} \cdot \frac{N}{a}(\mathrm{eV}) .
$$

For example, for a nano-Coulomb bunch $\left(N \sim 10^{10}\right)$, we have $\delta K_{\max } \sim 1 \mathrm{KeV}$ if $a \sim 1 \mathrm{~cm}$ and $\delta K_{\max } \sim$ $100 \mathrm{KeV}$ if $a \sim 100 \mu \mathrm{m}$. Naturally, for modern scientific accelerators, this value is relatively small. However, the effect of self-acceleration can be of practical importance for applications in the areas of industrial and medical accelerators.

\section{ACKNOWLEDGMENTS}

This research was supported by the grant of Saint Petersburg State University.

[1] V. L. Ginzburg and V. N. Tsytovich, Transition Radiation and Transition Scattering (Taylor \& Francis, London, 1990).

[2] S. A. Kheifets and S. A. Heifets, Report No. SLAC-PUB3965, 1986.

[3] L. Palumbo, Part. Accel. 25, 201 (1990).

[4] L. Palumbo, V. G. Vaccaro, and M. Zobov, Wake Fields and Impedance (CAS Advanced School on Accelerator Physics, Rhodes, Greece, 1994).

[5] T. Yu. Alekhina and A. V. Tyukhtin, Phys. Rev. ST Accel. Beams 16, 081301 (2013).

[6] B. M. Bolotovskii and G. V. Voskresenskii, Sov. Phys. Usp. 9, 73 (1966).

[7] V. Bobrinev and V. Braginskiy, Doklady AN USSR 123, 634 (1958).

[8] T. Yu. Alekhina and A. V. Tyukhtin, Phys. Rev. E 83, 066401 (2011).

[9] T. Yu. Alekhina and A. V. Tyukhtin, Phys. Rev. ST Accel. Beams 15, 091302 (2012).

[10] V.P. Zrelov, Cherenkov Radiation in High-Energy Physics (Program for Scientific Translations, Jerusalem, Israel, 1970).

[11] B. Noble, Methods Based on the Wiener-Hopf Technique for the Solution of Partial Differential Equations (Pergamon Press, London-New York-Paris-Los Angeles, 1958).

[12] L.A. Vainshtein, The Theory of Diffraction and the Factorization Method (Generalized Wiener-Hopf Technique) (Golem Press, Boulder, CO, 1969). 